\title{
Epidemiological and phylogenetic analysis of canine kobuviruses in Tangshan, China
}

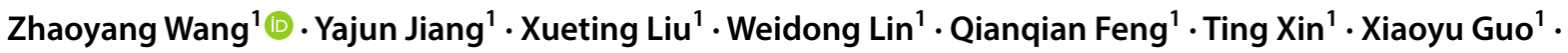 \\ Shaohua Hou ${ }^{1} \cdot$ Hongfei Zhu ${ }^{1} \cdot$ Hong Jia $^{1}$
}

Received: 15 February 2020 / Accepted: 4 June 2020 / Published online: 8 July 2020

(c) Springer-Verlag GmbH Austria, part of Springer Nature 2020

\begin{abstract}
Canine kobuviruses $(\mathrm{CaKoV})$ have been found in healthy and diarrheic dogs as well as asymptomatic wild carnivores in various countries. In order to investigate the prevalence and evolution of $\mathrm{CaKoV}$ in Tangshan, China, $82 \mathrm{dog}$ fecal samples from pet hospitals in Tangshan were subjected to RT-PCR targeting a segment of the 3D gene of CaKoV. Using this method, we identified CaKoV in 14 samples $(17.07 \%, 14 / 82)$. Of the CaKoV-positive samples, 78.57\% (11/14) and 50\% (7/14) were positive for canine parvovirus and canine coronavirus, respectively. The nucleotide sequences of the 14 strains $96.6 \%-100 \%$ identical to each other and 77.6\%-99.2\% identical to representative sequences from the NCBI GenBank database. We also amplified the 14 VP1 gene sequences and found that they were $93.3 \%-99.6 \%$ identical to each other and $73.3 \%-97.8 \%$ identical to representative sequences from the NCBI GenBank database. Phylogenetic analysis revealed that the $14 \mathrm{CaKoV}$ strains from Tangshan are closely related to those identified in China and Thailand and display less similarity to those found in Africa, the United States, and Europe. Our data suggest that CaKoV circulated in young pet dogs in Tangshan and displays a high co-infection rate with $\mathrm{CCoV}$ and CPV. However, the relationship between the three viruses and their roles in the host requires further investigation.
\end{abstract}

Keywords Canine kobuvirus $\cdot$ Epidemiology $\cdot$ Phylogenetic analysis $\cdot$ Molecular characterization

\section{Introduction}

Gastroenteritis is a common cause of hospitalization of dogs, and it primarily affects puppies under 1 year of age [1]. Due to the various causes of gastroenteritis, and particularly because coinfections with different viruses or bacteria are common, it can be challenging for veterinarians to determine the etiological agent causing the disease [2, 3]. Kobuviruses have been shown to be potential gastroenteritis-causing agents in dogs [4].

The genus Kobuvirus family Picornaviridae includes members of the species Aichivirus A-F and two unclassified viruses, according to the International Committee on

Handling Editor: Ana Cristina Bratanich.

Hong Jia

jiahong80@126.com

1 Institute of Animal Sciences, Chinese Academy of Agricultural Sciences, No. 2 Yuanmingyuan West Road, Haidian, Beijing 100193, China
Taxonomy of Viruses (ICTV) reports [5-8]. Canine kobuvirus $(\mathrm{CaKoV})$ is a member of the species Aichivirus $A$ [9]. The CaKoV genome is $8.1-8.2 \mathrm{~kb}$ in length, with a $5^{\prime}$ untranslated region (UTR), an open reading frame (ORF), and a 3'UTR [9]. The ORF is 7,332-7,341 nucleotides (nt) in length and encodes a single polyprotein, which is separated into a leader (L) protein, three structural proteins, and seven non-structural proteins $[4,10]$.

$\mathrm{CaKoV}$ was first detected in diarrheic dogs in the United States in 2011 [4, 10]. To date, CaKoV has also been detected in both healthy and diarrheic dogs in the United Kingdom [11], Italy [12], Korea [13], Tanzania [14], Japan [15], China [16, 17], Thailand [18], and Brazil [19]. In this study, we performed an analysis of partial 3D and VP1 gene sequences in order to compare the strains we detected with $\mathrm{CaKoV}$ strains reported elsewhere to determine the prevalence of $\mathrm{CaKoV}$ in Tangshan, China, and to improve our understanding of the relationship between different viruses and hosts. 


\section{Materials and methods}

\section{Sampling}

Eighty-two fecal samples were provided by pet hospitals in Tangshan between April and June 2019. Of the samples, 62 samples were from animals with diarrhea. All samples were stored in ultra-low-temperature freezers $\left(-80^{\circ} \mathrm{C}\right)$.

\section{DNA and RNA extraction}

The fecal samples were dissolved in phosphate-buffered saline (PBS), and the supernatant was collected by centrifugation. DNA and RNA were extracted from the supernatant using an AxyPrep ${ }^{\mathrm{TM}}$ Body Fluid Viral DNA/RNA Miniprep Kit (Corning Inc., New York, USA). Extracted RNA was reverse transcribed into cDNA using an M5 HiPer First Strand cDNA Synthesis Kit (Me5 Technology Co., Ltd., Beijing, China).

\section{Detection of pathogens}

All fecal samples were tested for canine parvovirus 2 (CPV2 ), canine coronavirus ( $\mathrm{CCoV})$, and $\mathrm{CaKoV}$ by PCR or RT-PCR according to previously described procedures [11, 20-22]. The purified amplification products of the $\mathrm{CaKoV}$ $3 \mathrm{D}$ gene fragment and the VP1 gene were ligated into a Blunt-Zero cloning vector using a 5 min TA/Blunt-Zero Cloning Kit (Vazyme Biotech Co., Ltd., Nanjing, China). Recombinant Blunt-Zero cloning vectors were introduced into DH5 $\alpha$ competent cells by transformation, and individual colonies were picked three times for sequencing using the Sanger method. Fourteen partial sequences of the 3D and VP1 genes of CaKoV were deposited in the GenBank (NCBI) database (accession nos. MT0199770-MT019983 and MT465833-MT465846).

\section{Phylogenetic analysis of 3D and VP1 genes}

For phylogenetic analysis, partial sequences of CaKoV 3D and VP1 genes from canines were retrieved from the GenBank database for use as reference sequences. The ClustalX program was used to make multiple alignments of the sequences [23], and MEGA 6.06 [24] was used to generate a phylogenetic tree, by neighbor-joining (NJ) method with 1000 bootstrap replicates.

\section{Results}

We detected $10(16.13 \%, 10 / 62) \mathrm{CaKoV}$-positive samples from 62 diarrheic dogs and 4 (20.00\%, 4/20) CaKoV-positive samples from 20 dogs without diarrhea. Thus, the overall rate of $\mathrm{CaKoV}$ positivity in our sample population was $17.07 \%$ (14/82). The characteristics of the CaKoV-positive dogs are presented in Table 1, and age information about the dogs used in this study is presented in Table 2. Of the 82 samples, CPV-2 was detected in 64 (78.04\%) and CCoV was detected in 14 (17.07\%). Of the CaKoV-positive samples, $78.57 \%$ (11/14) were co-infected with CPV-2, 50\% (7/14) were co-infected with $\mathrm{CCoV}$, and $42.86 \%$ (6/14) were coinfected with both CPV-2 and CCoV I/II.
Table 1 Fecal samples from Tangshan, China, that were positive for canine kobuvirus

\begin{tabular}{llllllll}
\hline Number & Dog identification & Age & Gender & Diarrhea & $\begin{array}{l}\text { CaKoV } \\
\text { partial 3D } \\
\text { gene }\end{array}$ & CPV/subtype & CCoV/subtype \\
& & & & & \\
\hline 1 & TS-A3 & 3 months & F & No & + & $+/ 2 \mathrm{c}$ & - \\
2 & TS-A4 & 2 months & M & Yes & + & - & - \\
3 & TS-B4 & 4 months & M & Yes & + & $+/ 2 \mathrm{c}$ & $+/$ II \\
4 & TS-C2 & 2 months & M & Yes & + & - & - \\
5 & TS-C5 & 1 month & F & No & + & - & $+/$ II \\
6 & TS-D18 & 4 months & M & Yes & + & $+/ 2 \mathrm{c}$ & $+/$ II \\
7 & TS-E1 & 3 months & F & Yes & + & $+/ 2 \mathrm{c}$ & $+/$ II \\
8 & TS-E4 & 2 months & F & Yes & + & $+/ 2 \mathrm{c}$ & $+/$ I \\
9 & TS-G3 & 2 months & M & Yes & + & $+/ 2 \mathrm{c}$ & - \\
10 & TS-G8 & 3 months & M & Yes & + & $+/ 2 \mathrm{a} / 2 \mathrm{c}$ & $+/$ II \\
11 & TS-H5 & 2 months & F & Yes & + & $+/ 2 \mathrm{c}$ & - \\
12 & TS-H7 & 2 months & M & No & + & $+/ 2 \mathrm{c}$ & - \\
13 & TS-H8 & 2 months & M & No & + & $+/ 2 \mathrm{c}$ & $+/$ II \\
14 & TS-I6 & 3 months & F & Yes & + & $+/ 2 \mathrm{c}$ & - \\
\hline
\end{tabular}

+ , positive; -, negative; M, male; F, female 
Table 2 Age characteristics of the dogs used in this study

\begin{tabular}{llll}
\hline Age & \multicolumn{2}{l}{ CaKoV positive $(\%)$} & \multirow{2}{*}{ Total } \\
\cline { 2 - 3 } & Asymptomatic & Diarrhea & \\
\hline Young (<1 year) & $4 / 16(25.00 \%)$ & $10 / 54(18.52 \%)$ & $14 / 70(20.00 \%)$ \\
Adult (1-5 years) & $0 / 4(0 \%)$ & $0 / 7(0 \%)$ & $0 / 11(0 \%)$ \\
Older (>5 years) & $0(0 \%)$ & $0 / 1(0 \%)$ & $0 / 1(0 \%)$ \\
& $4 / 20(20.00 \%)$ & $10 / 62(16.13 \%)$ & \\
\hline
\end{tabular}

Phylogenetic analysis (NJ) of the 14 partial 3D gene sequences and reference sequences was performed using MEGA 6 software with 1000 bootstrap replicates (Fig. 1). The 14 sequences shared $96.6 \%-100.0 \%$ nt sequence identity with each other and $77.6 \%-99.2 \%$ nt sequence identity with other previously reported representative $\mathrm{CaKoV}$ sequences. The TS-C2, TS-G8, TS-A3, and Gifu0226 strains formed a small cluster, with nt sequence identity between $98.2 \%$ and $100.0 \%$. TS-G3 was located between two small branches and exhibited the closest relationship to strain SMCQ-S4 (98.4\% nt sequence identity). Strain TS-E1 was most closely related to strain UK003 from the United Kingdom (96.6\% nt sequence identity). The strains TS-H8, TS-C5, TS-I6, and SMCQ-M9 formed a small branch with $97.8 \%-98.6 \%$ nt sequence identity. The strains TS-A4 and TS-B4, form an independent small branch with the Tokyo1173 strain and the CU_125 strain from Thailand with $97.4 \%-99.8 \%$ nt sequence identity. The highest nt sequence identity was observed between the strains TS-H5 and SMCQ-M20 (99.2\%). The strains TS-E4, TS-D18, TS-H7, and Y12 form a small branch with 98.2\%-99.0\% nt sequence identity.

Phylogenetic analysis was also performed as described above using VPI gene sequences (Fig. 2). The 14 sequences shared $93.3 \%-99.6 \%$ nt sequence identity with each other and $73.3 \%-97.8 \%$ nt identity with other previously reported representative sequences. The strains CM-G8, CM-H8, CU_53, CU_83, CU_101, and CU_273 formed a small cluster with $96.8 \%-99.5 \%$ nt sequence identity. The strains CM-A3, CM-E4, CM-C2, CM-I6, and AH-1 formed a small cluster with $97.7 \%-98.7 \%$ nt sequence identity. CM-E1 was located between two small branches and displayed the closest relationship to strains SMCD-59 and SMCD-M9 (97.0\% nt sequence identity). Strains CM-B4, CM-H7, CM-D18, and CM-H5 formed a small branch with $96.0 \%-99.6 \% \mathrm{nt}$ sequence identity. CM-G3 was located between two small branches and displayed the closest relationship to strain HLJ2015-7 (94.9\% nt sequence identity). The strains CM-A4, CM-C5, and HLJ2015-7 formed a small branch with $95.8 \%-98.3 \%$ nt sequence identity. Overall, the 14 sequences from our study samples, nine sequences from other regions of China, and 12 sequences from Thailand formed an independent larger branch.

\section{Discussion}

The CaKoV-positive rate observed in this study (16.13\%) was similar to those reported previously in diarrheic dogs in northeast China (17.91\%) [17], South Korea (19.0\%) [25], and Thailand (17.59\%) [18] and lower than those reported in southwest China (50.46\%) [22] and Japan (37.2\%) [15]. The CaKoV-positive rates in diarrheic dogs observed in the United States, Italy, and Brazil were lower (3\%, $4.4 \%$, and $5.7 \%$, respectively) than that observed in our study $[4,12,19]$. These data indicate that CaKoVpositive rates may differ between different countries or regions. Some studies have found $\mathrm{CaKoV}$ not only in dogs with gastroenteritis but also in healthy animals $[15,18,22$, 25]. In this study, CaKoV was detected in $20 \%$ (4/20) of dogs without diarrhea symptoms. These results indicate that $\mathrm{CaKoV}$ is present in both healthy and diarrheic dogs. However, the relationship between the virus and diarrhea remains unclear and requires further study.

In this study, all CaKoV-positive dogs were between 1 and 4 months old, similar to the ages of those in the report by Soma et al. [15]. However, Miyabe et al. [19] detected $\mathrm{CaKoV}$ in 2-year-old adult dogs. Further analysis by Charoenkul et al. [18] showed that the infection rates of puppies ( $<1$ year) and old dogs ( $>5$ years) are similar (25.45\% and $18.18 \%$, respectively) and are much higher than those in adult dogs in the 1-5 year age group (4.08\%). This indicates that $\mathrm{CaKoV}$ can infect dogs of all ages and that puppies and elderly dogs may be infected at higher rates. Diarrhea in dogs is often caused by simultaneous infection with viruses, bacteria, and parasites as well as multiple viruses [1, 26, 27]. In order to detect coinfections with $\mathrm{CCoV}$ and $\mathrm{CPV}$, we tested for the presence of the $\mathrm{S}$ gene of $\mathrm{CCoV}$ and the VP2 gene of CPV in the $\mathrm{CaKoV}$-positive materials. The $\mathrm{CaKoV}$-CCoV coinfection rate was $50 \%$ (7/14); CCoV I and CCov II were found in $7.14 \%(1 / 14)$ and $42.86 \%$ (6/14) of these coinfections, respectively. The $\mathrm{CaKoV}-\mathrm{CPV}$ coinfection rate was $78.57 \%$ (11/14); most of these coinfections were with CPV $2 \mathrm{c}$ subtypes. Interestingly, a mixed CPV subtype $2 \mathrm{a}$ and $2 \mathrm{c}$ infection was detected in one sample (sample no. 10, Table 1). One sample was positive for $\mathrm{CaKoV}$, but not for $\mathrm{CPV}$ or $\mathrm{CCoV}$, suggesting that $\mathrm{CaKoV}$ alone may cause diarrhea, which is consistent with findings from northeastern China [17].

In order to investigate the genetic relationship between CaKoV strains in dogs in Tangshan, China, partial 3D and VP1 gene sequences of the $14 \mathrm{CaKoV}$-positive strains were compared and used for phylogenetic analysis. The VP1 gene encodes the kobuvirus capsid protein, which is the most variable gene among kobuviruses $[28,29]$. The 14 VP1 sequences formed a large branch with previously 


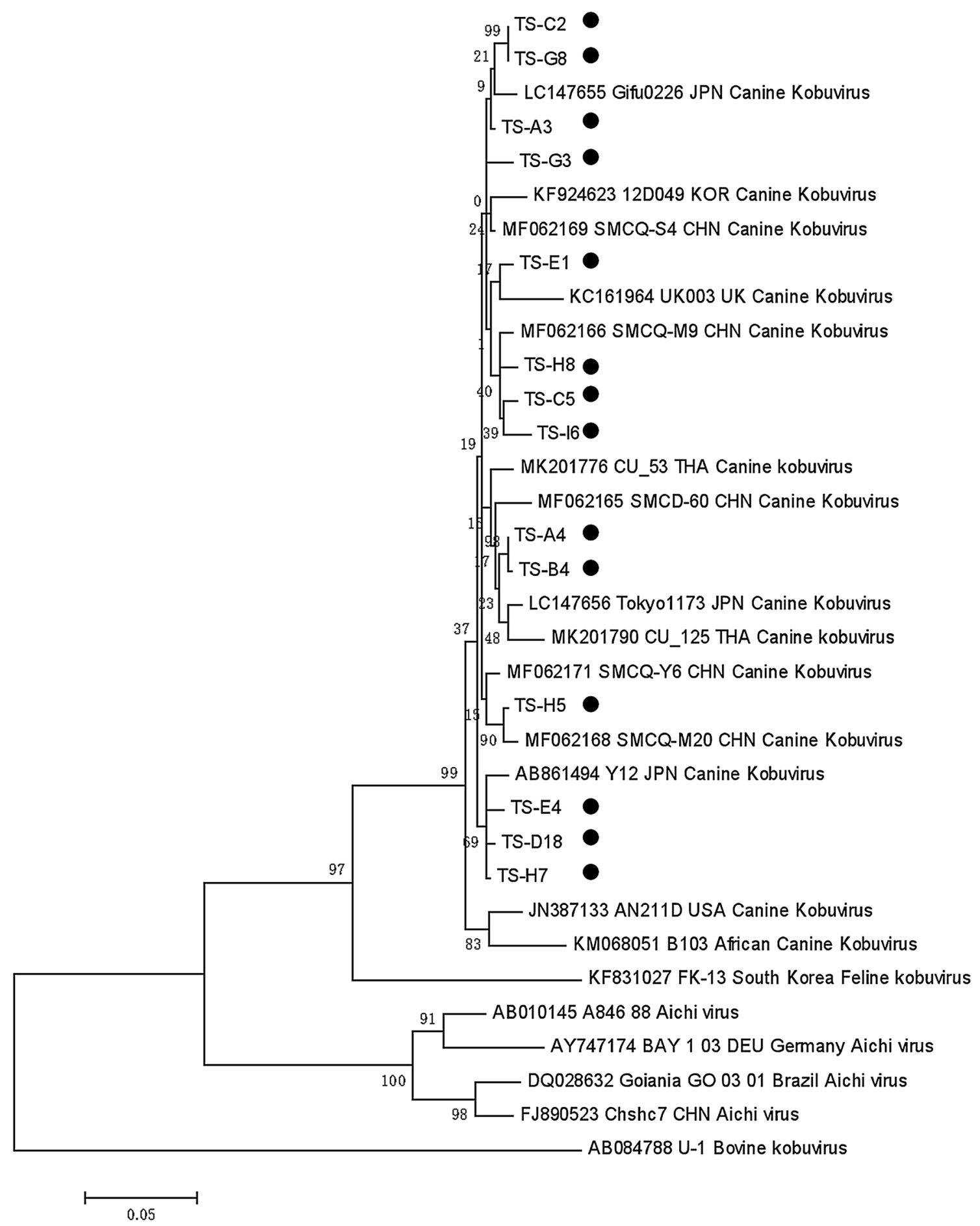

Fig. 1 Phylogenetic tree based on partial 3D gene sequences (504 bp) from CaKoVs, constructed using the neighbor-joining (NJ) method in MEGA 6 software. Bootstrap analysis was performed with 1,000 replicates. $\bullet$ represents the CaKoV strains identified in this study

published CaKoV sequences from China and Thailand, which is consistent with previous research and indicates that kobuvirus strains may have strong geographical distributions [18].
Fig. 2 Phylogenetic tree based on VP1 gene sequences (834 bp) from CaKoVs, constructed using the neighbor-joining (NJ) method in MEGA 6 software. Bootstrap analysis was performed with 1,000 replicates. represents the $\mathrm{CaKoV}$ strains identified in this study 


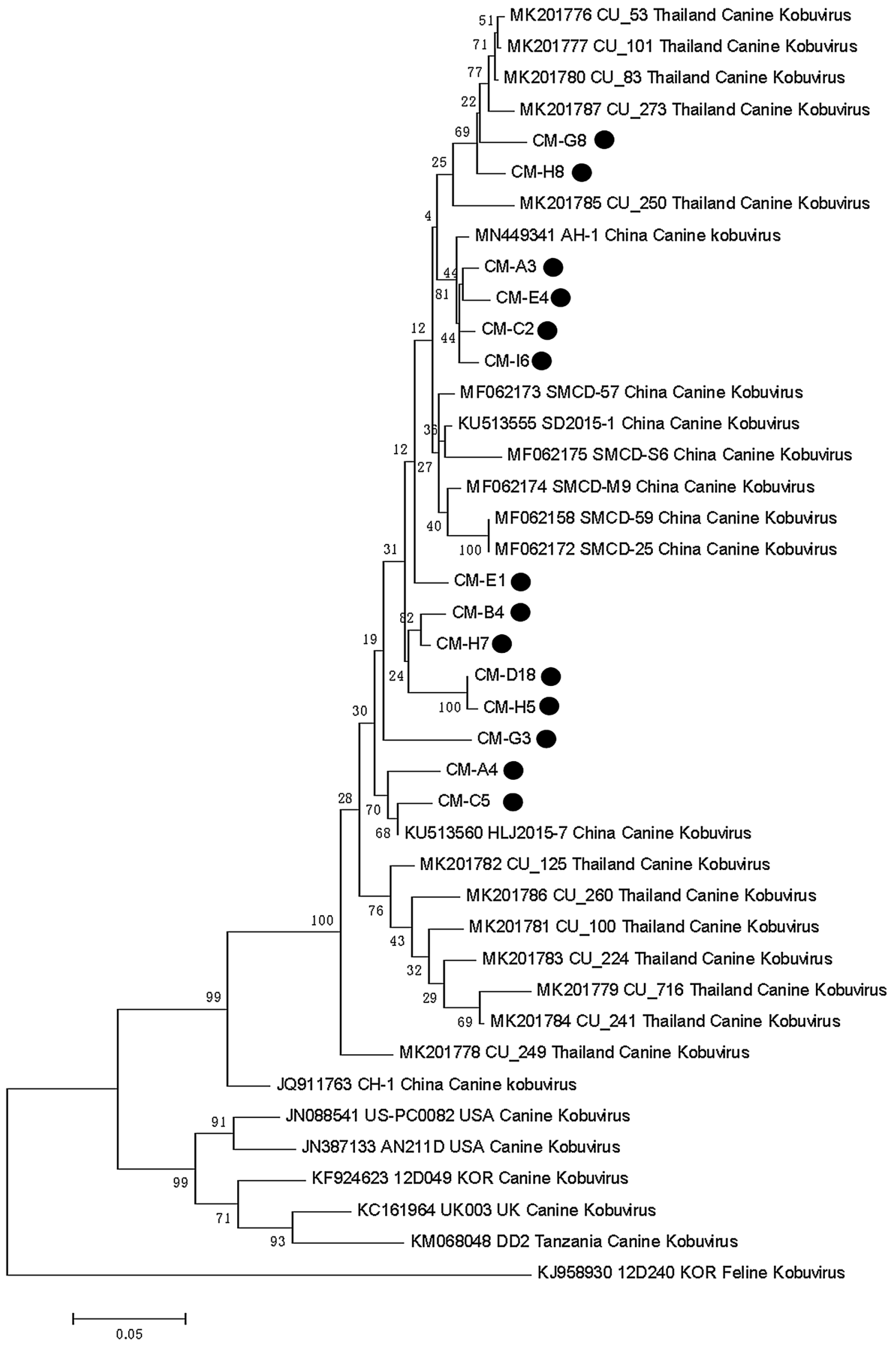


In conclusion, we report for the first time an epidemiological and phylogenetic analysis of canine kobuvirus (CaKoV) in Tangshan, China. Phylogenetic analysis showed that the distribution of the virus displays regional differences. Compared with other enteroviruses, the strain of the $\mathrm{CaKoV}$ virus in Tangshan has greater genetic diversity and a higher coinfection rate. Virus positivity appears to be associated with age; puppies and elderly dogs may be infected at a higher rate than adult dogs.

Funding This study was funded by the National Key Research and Development Program of China (2016YFD0501003).

\section{Compliance with ethical standards}

Conflict of interest The authors declare that they have no conflicts of interest.

Ethical approval All applicable international, national, and/or institutional guidelines for the care and use of animals were followed. Animal experiments (sampling) were approved by the Animal Care and Use Committee of the Institute of Animal Sciences of the Chinese Academy of Agricultural Sciences. Sampling and data release were approved by the animals' owners.

\section{References}

1. Ortega AF, Martinez-Castaneda JS, Bautista-Gomez LG et al (2017) Identification of co-infection by rotavirus and parvovirus in dogs with gastroenteritis in Mexico. Braz J Microbiol 48(4):769-773

2. Gizzi AB, Oliveira ST, Leutenegger CM et al (2014) Presence of infectious agents and co-infections in diarrheic dogs determined with a real-time polymerase chain reaction-based panel. BMC Vet Res 10:23

3. Martella V, Pratelli A, Elia G et al (2001) Isolation and genetic characterization of two G3P5A canine rotavirus strains in Italy. J Virol Methods 96(1):43-49

4. Li L, Pesavento PA, Shan T et al (2011) Viruses in diarrheic dogs include novel kobuviruses and sapoviruses. J Gen Virol 92(Pt 11):2534-2541

5. Adams MJ, Lefkowitz EJ, King AM et al (2016) Ratification vote on taxonomic proposals to the International Committee on Taxonomy of Viruses. Arch Virol 161(10):2921-2949

6. Oem JK, Lee MH, Lee KK et al (2014) Novel Kobuvirus species identified from black goat with diarrhea. Vet Microbiol 172(3-4):563-567

7. Yamashita T, Ito M, Kabashima Y et al (2003) Isolation and characterization of a new species of kobuvirus associated with cattle. J Gen Virol 84(Pt 11):3069-3077

8. Gang L, Xin Z, Jie L et al (2018) First report and genetic characterization of feline kobuvirus in diarrhoeic cats in China. Transbound Emerg Dis 65:1357-1363

9. Khamrin P, Maneekarn N, Okitsu S et al (2014) Epidemiology of human and animal kobuviruses. Virus Dis 25(2):195-200

10. Kapoor A, Simmonds P, Dubovi EJ et al (2011) Characterization of a canine homolog of human Aichivirus. J Virol 85(21):11520-11525
11. Carmona-Vicente N, Buesa J, Brown PA et al (2013) Phylogeny and prevalence of kobuviruses in dogs and cats in the UK. Vet Microbiol 164(3-4):246-252

12. Di Martino B, Di Felice E, Ceci C et al (2013) Canine kobuviruses in diarrhoeic dogs in Italy. Vet Microbiol 166(1-2):246-249

13. Choi S, Lim SI, Kim YK et al (2014) Phylogenetic analysis of astrovirus and kobuvirus in Korean dogs. J Vet Med Sci 76(8):1141-1145

14. Olarte-Castillo XA, Heeger F, Mazzoni CJ et al (2015) Molecular characterization of canine kobuvirus in wild carnivores and the domestic dog in Africa. Virology 477:89-97

15. Soma T, Matsubayashi M, Sasai K (2016) Detection of kobuvirus RNA in Japanese domestic dogs. J Vet Med Sci 78(11):1731-1735

16. Kong N, Zuo Y, Wang Z et al (2016) Molecular characterization of new described kobuvirus in dogs with diarrhea in China. Springerplus 5(1):2047

17. Li C, Wei S, Guo D et al (2016) Prevalence and phylogenetic analysis of canine kobuviruses in diarrhoetic dogs in northeast China. J Vet Med Sci 78(1):7-11

18. Charoenkul K, Janetanakit T, Chaiyawong S et al (2019) First detection and genetic characterization of canine Kobuvirus in domestic dogs in Thailand. BMC Vet Res 15(1):254

19. Miyabe FM, Ribeiro J, Alfieri AF et al (2019) Detection of canine kobuvirus RNA in diarrheic fecal samples of dogs with parvoviruses. Braz J Microbiol 50(3):871-874

20. Wang X, Li C, Guo D et al (2016) Co-circulation of Canine Coronavirus I and II $\mathrm{a} / \mathrm{b}$ with high prevalence and genetic diversity in Heilongjiang Province Northeast China. PLoS ONE 11(1):e146975

21. Buonavoglia D, Cavalli A, Pratelli A et al (2000) Antigenic analysis of canine parvovirus strains isolated in Italy. New Microbiol 23(1):93-96

22. Li M, Yan N, Wang M et al (2018) Prevalence and genomic characteristics of canine kobuvirus in southwest China. Arch Virol 163(2):459-466

23. Thompson JD, Gibson TJ, Plewniak F et al (1997) The CLUSTAL_X windows interface: flexible strategies for multiple sequence alignment aided by quality analysis tools. Nucleic Acids Res 25(24):4876-4882

24. Tamura K, Stecher G, Peterson D et al (2013) MEGA6: molecular evolutionary genetics analysis version 6.0[J]. Mol Biol Evol 30(12):2725-2729

25. Oem JK, Choi JW, Lee MH et al (2014) Canine kobuvirus infections in Korean dogs. Arch Virol 159(10):2751-2755

26. Duijvestijn M, Mughini-Gras L, Schuurman N et al (2016) Enteropathogen infections in canine puppies: (Co-)occurrence, clinical relevance and risk factors. Vet Microbiol 195:115-122

27. Headley SA, Alfieri AA, Fritzen JT et al (2013) Concomitant canine distemper, infectious canine hepatitis, canine parvoviral enteritis, canine infectious tracheobronchitis, and toxoplasmosis in a puppy. J Vet Diagn Invest 25(1):129-135

28. Reuter G, Boros A, Pankovics (2011) Kobuviruses-a comprehensive review. Rev Med Virol 21(1):32-41

29. Yamashita T, Ito M, Kabashima $Y$ et al (2003) Isolation and characterization of a new species of kobuvirus associated with cattle. J Gen Virol 84:3069-3077

Publisher's Note Springer Nature remains neutral with regard to jurisdictional claims in published maps and institutional affiliations. 